\title{
Water Triggered Shape Memory Materials
}

\author{
Guoguang Niu ${ }^{*}$ and Daniel Cohn $\dagger$
}

\begin{abstract}
SUMMARY: THE SHAPE MEMORY materials triggered by water display advantage over the thermal induced ones because water triggered can eliminate temperature constrains and avoids fast degradation. Here we reported a new strategy of water induced shape memory, in which the formation and dissolution of poly(ethylene glycol) crystal is utilized for the fixation and recovery of temporary deformation of hydrophilic polymer. This water-induced shape recovery is less sensitive to temperature, of which $95 \%$ deformation is fixed in circumstance and over $75 \%$ recovery is reached even at $0{ }^{\circ} \mathrm{C}$. The material possesses potential applications in biomedical fields, such as artificial lens and smart suture.
\end{abstract}

*: Institute of Regenerative Medicine, Wake Forest University, Winston-Salem, NC 27103, USA

$\dagger$ : Casali Institute of Applied Chemistry, Institute of Chemistry, The Hebrew University of Jerusalem, Jerusalem 91904, Israel

$\Delta$ : Correspondence to: Dr. Guoguang Niu, 391 Technology Way, Winston-Salem, NC 27101, USA

Tel: +1-336-776-8748

Email: guoguang.niu@gmail.com

Received: July 19, 2013

Revised: July 31, 2013

Accepted: August 02, 2013

SCIENCE INSIGHTS 2013; 3(1):49-50.

Keywords: Shape memory - Biodegradable - Biomaterials - Poly(ethylene glycol) - Crystal

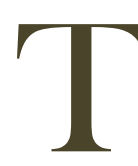

$\checkmark$ HE TERM "shape memory effect" refers to the ability of a material to be deformed and fixed into a temporary shape, and to recover its original, permanent shape upon an external stimulus (1). Shape memory polymers have attracted much interest because of their unique properties, and applied tremendously in medical area, such as biodegradable sutures, actuators, catheters and smart stents $(2,3)$. Shape memory usually is a thermally induced process, although it can be activated by light illumination, electrical current, magnetic, or electromagnetic field (4-6). During the process, the materials are heated directly or indirectly above their glass transition temperature $\left(\mathrm{T}_{\mathrm{g}}\right)$ or the melting temperature $\left(\mathrm{T}_{\mathrm{m}}\right)$ in order to recover the original shape. Nonthermally induced shape memory polymers eliminate the temperature constrains and enable the manipulation of the shape recovered under ambient temperature $(7,8)$.
Herein, we report a novel strategy of water induced shape memory, in which the formation and dissolution of poly(ethylene glycol) (PEG)

crystal is utilized for the fixation and recovery of temporary deformation of hydrophilic polymer. This waterinduced shape recovery is less sensitive to temperature, of which $95 \%$ deformation is fixed in circumstance and over $75 \%$ recovery is reached even at $0{ }^{\circ} \mathrm{C}$.

Water-driving - shape recovery at ambient temperature has been described (9), which is dependent on the decrease of $T_{\mathrm{g}}$ or the dissolution of PEG segment. However, the recovery rate of these materials also depends on temperature, $70 \%$ shape recovery requiring at least $30{ }^{\circ} \mathrm{C}$ or even higher temperatures. For some hydrophilic polymers, the samples behave like crystalline materials without water, in which a linear stress-strain relation can be found prior to yielding (10). While with the uptake of water, the behavior of these materials will change to rubbers.
Based on this transition, we developed one kind of water induced shape memory materials. This kind of material contains hydrophobic poly ( $\varepsilon$-caprolactone) (PCL) or poly(L- lactide) (PLA) segment and PEG segment. PLA or PCL segments bestow the materials the biodegradable properties in physiological conditions. PEG segments are prone to forming crystals in dried materials (Fig. 1A). However when the materials are swollen in water, the PEG crystalline structure is destroyed, and the rubber-like materials are formed (Fig. 1B). These swollen samples can be deformed into different shapes (Fig. 1C). After drying, the PEG crystalline structure restored again, as thus the predetermined shapes are fixed (Fig.1D). These new shapes are stable for a long time at room temperature, and they can recover their original shapes with the disappearance of PEG crystals when immersed into water.

The ability of polymers to form different predetermined temporary 
A
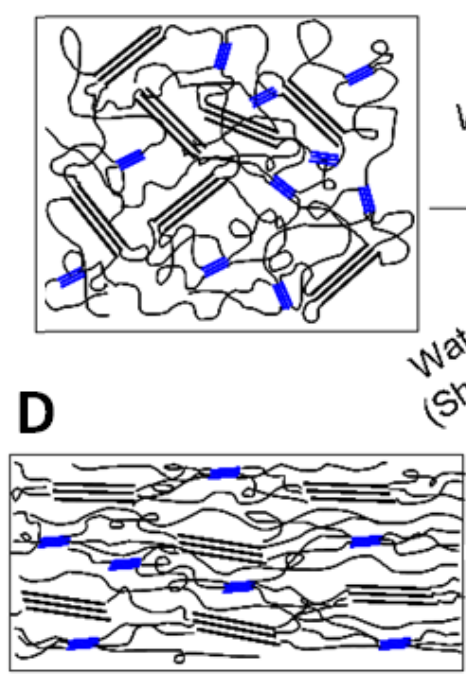

戸 : PEG crystal
B

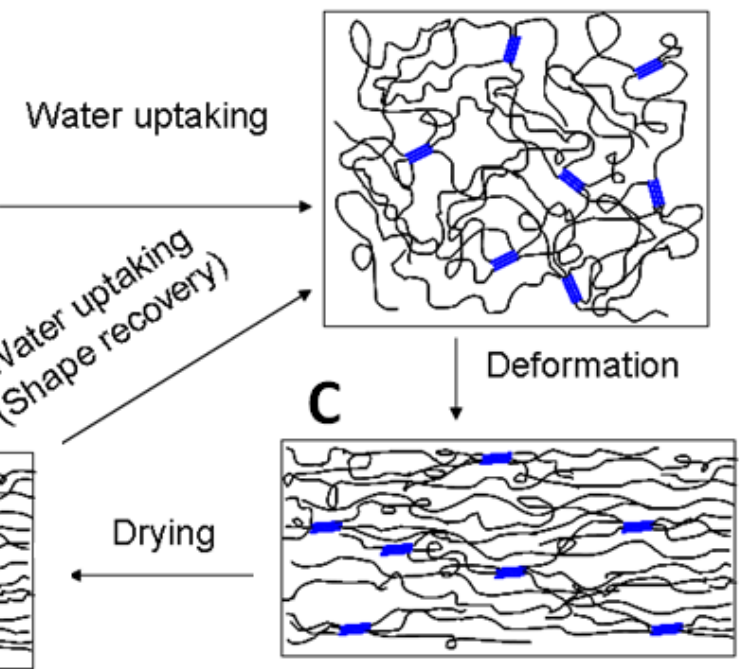

$\equiv$ : PLA or PCL

Figure 1. Schematic of water triggered shape memory and restoration. With the uptake of water PEG crystals in material (A) transform into amorphous state (B); and the wet material is easily elongated or deformed (C); after drying, PEG crystals restored and the deformed shape is fixed or memorized (D); with water uptake the reformed material returns back its initial shape..

shapes and subsequently recover their initial shapes within a wide temperature range by water uptake eliminate the limitation of temperature during the stages of fixation and recovery that enables the materials to be used widely in biomedical fields, such as self-tightening suture, artificial lens, smart stents, and artificial scaffold with complex geometries.

\section{Conflict of Interests}

None

\section{List of Abbreviations}

PEG: poly(ethylene glycol); $T_{g}$ : glass transition temperature; $\mathrm{T}_{\mathrm{m}}$ : melting temperature; PCL: poly $(\varepsilon-$ caprolactone); PLA: poly(L- lactide)

\section{References}

1. Osada Y, Matsuda A. Shape memory in hydrogels. Nature 1995; 376: 219.

2. Lendlein A, Langer R. Biodegradable elastic shape-memory polymers for potential biomedical applications. Science 2002; 296: 1673-6.

3. Gall K, Yakacki CM, Liu Y, Shandas R, Willett N, Anseth KS. Thermomechanics of the shape memory effect in polymers for biomedical applications. J Biomed Mater Res Part A 2005; 73: 339-48.

4. Koerner H, Price G, Pearce NA, Alexander M, Vaia RA. Remotely actuated polymer nanocompositesstress-recovery of carbonnanotube-filled thermoplastic elastomers. Nat Mater 2004; 3: 115-20.

5. Vaia R. Nanocomposites: remotecontrolled actuators. Nat Mater 2005; 4: 429-30.

6. Razzaq MY, Anhalt M, Frormann L, Weidenfeller B. Thermal electrical and magnetic studies of magnetite filled polyurethane shape memory polymers. Mater Sci Eng A 2007; 444: 227-35.

7. Palffy-Muhoray P, CamanchoLopez M, Finkelmann H, Shelley M. Fast liquid crystal elastomer swins into the dark. Nat Mater 2004; 3: 307-10.

8. Gupta MC and Deshmukh VG. Thermal oxidative degradation of poly-lactic acid Part II: molecular weight and electronic spectra during isothermal heating. Colloid \& Polymer Sci 1982; 260: 514-7.

9. Huang WM, Yang B. Water-driven programmable polyurethane shape memory polymer: demonstration and mechanism. Applied Physics Letter 2005; 86: 114105.

10. Yang B, Huang WM, Li C, Li L. Effects of moisture on the thermomechanical properties of a polyurethane shape memory polymer. Polymer 2006; 47: 1348-56.

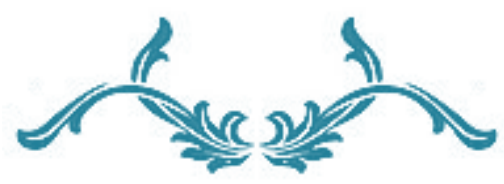

